\title{
Analysis of a distributed MIMO channel capacity under a special scenario
}

\author{
Xiaonan Zhao ${ }^{1,2^{*}}$ (D) Pengzhen $\mathrm{Jia}^{3}$, Qianwen Zhang ${ }^{4}$, Yupeng Li ${ }^{1,2}$, Ruiqing Xing ${ }^{1,2}$ and Yuan Liu ${ }^{1,2}$
}

\begin{abstract}
A distributed antenna system enhances the coverage performance of a system by distributing the antennas geographically, as well as by improving the diversity gain and capacity performance. The channel impulse response matrix of a distributed MIMO system in an airplane cabin environment is measured by a self-built channel measurement platform. The transmitting antennas are distributed, and the receiving antennas are placed centrally. The data analysis shows that the distributed MIMO system can significantly increase the system's channel capacity. The system's capacity is also closely related to whether the antennas are evenly placed. When the antennas are evenly placed, the capacity is increased and the distribution is more uniform.
\end{abstract}

Keywords: Communication channels, Modeling, MIMO, Channel capacity

\section{Introduction}

Network technology has achieved a state of rapid development, and an increasing number of people are paying attention to the development of communication technology. China has achieved diversified and multi-angle development in communication technology. The emergence of $5 \mathrm{G}$ technology will surely set off a wave of new networks, as 5G technology can not only meet the needs of consumers in terms of speed, but it can also achieve higher levels of efficiency, which is a driving force for the future development of society. In the current situation, many young people are paying more attention to the pursuit of high-tech technology, and an increasing number of people have chosen more diversified network systems. The development and progression of Internet communication technology represent not only the development of science and technology, but also the progress of the national economy [1-3].

Multi-antenna technology, which is the mainstream alternative technology for next-generation wireless communication, can greatly improve the capacity and spectrum utilization efficiency of wireless systems. Since the introduction of MIMO technology, theoretical research on MIMO

\footnotetext{
* Correspondence: xiaonan5875@163.com

${ }^{1}$ Tianjin Key Laboratory of Wireless Mobile Communications and Power

Transmission, Tianjin Normal University, Tianjin 300387, China

${ }^{2}$ College of Electronic and Communication Engineering, Tianjin Normal

University, Tianjin 300387, China

Full list of author information is available at the end of the article
}

has covered various aspects, including system capacity, precoding technology, and receiver detection. In recent years, a variety of MIMO scenarios have been measured abroad [4-6], a large number of channel parameters have been obtained, and the corresponding channel models have been established. Domestic MIMO wireless channel measurements have also been performed [7-9]. The two outdoor channels are measured in different frequency bands, and the corresponding large-scale channel parameters have been obtained. This paper relies on the channel measurement platform built by Tsinghua University to measure the wireless channel of an indoor distributed antenna system and systematically analyzes its capacity.

Relying on the channel measurement platform independently developed by Tsinghua University, this paper measures the channel impulse response matrix of a distributed MIMO system in an MD-82 cabin environment. The measurement platform detects MIMO channels based on the timing of the transceiver antenna timing switching and supports up to 7 transmissions and 7 MIMO channel measurements. Based on the measured channel matrix in the cabin, the channel capacity of the distributed MIMO system and the SISO system is calculated and compared. 


\section{Methods/experimental}

\subsection{Measurement platform performance}

The measurement platform is a seven-transmitting-andseven-receiving system. The center frequency of the system is $3.52 \mathrm{GHz}$, and the bandwidth is $20 \mathrm{MHz}$. The specific parameters of the measurement platform are operating frequency $3.51 \sim 3.53 \mathrm{GHz}$, bandwidth $20 \mathrm{MHz}$, maximum transmit power value $20 \mathrm{dBmw}$, supported maximum Doppler bandwidth $797.2 \mathrm{~Hz}$, maximum travel speed $123 \mathrm{~km} / \mathrm{h}$, minimum resolution delay $50 \mathrm{~ns}$, maximum delay spread $12.8 \mu \mathrm{s}$, and an antenna and polarization omnidirectional antenna, with a $4 \mathrm{dBi}$ gain, and a vertical polarization.

\subsection{The system's working sequence}

Tx1 7 is the transmit antenna switching timing, $\mathrm{Rx} 1 \sim 7$ is the receiving antenna switching timing, and tp is the duration of the training sequence, as well as the value of the maximum delay spread. Here, $t p=12.8$ us is selected. The time for switching the antenna for 1 week is $7 \times 7 \times 25.6=1254.4$ us. Therefore, the maximum Doppler frequency that can be measured is $797.2 \mathrm{~Hz}$, which corresponds to a maximum moving speed of $123 \mathrm{~km} / \mathrm{h}$.

\subsection{Measurement environment introduction}

Since the originating antenna transmits signals by switching the same antenna through the switches, in order to make the characteristics of each antenna substantially similar, the seven antennas need to be equally spaced. The antennas are fixed to non-metal plates by coaxial cables, and then, the plates are placed on the top of a luggage cabinet. The spacing is the length of the two luggage cabinets, with the $\mathrm{Tx} 1 \sim 7$ at the center arrow position of the luggage rack at an interval of 290 $\mathrm{cm}$. Tx1 (the first antenna) is in the front section of the economy class, $159 \mathrm{~cm}$ away from the bulkheads of the economy and business class, and then, $\mathrm{Tx} 2 \sim \mathrm{Tx} 7$ are arranged linearly at equal intervals. The height of the bottom of the antenna (i.e., the fixed part without height) is $1.91 \mathrm{~m}$, at $0.53 \mathrm{~m}$ from the left side of the luggage rack and $0.56 \mathrm{~m}$ from the right side of the luggage rack. The receiving end receives with a linear array of 9 antennas (including 2 dummy antennas).

The table of the receiving antenna array is placed on the seat for a static fixed point measurement, and the reference direction of the receiving antenna array is directed towards the rear. The measurement sequence is from $\mathrm{E}$ to $\mathrm{A}$, and the measurement range is from the 4th to the 28th row of the economy class. Due to the limited cable length, measurements are not made in rows 29 to 33. Three groups were measured at each point, with each group being $4 \mathrm{~s}$ long. It should be noted that the small table in the fourth row (the first row of the economy class seats) is on the armrests on both sides of the seat, and the rests are in the backrest position of the seat. It should also be noted that there are only three seats (C, D, and E) for rows 26 to 28 .

\section{Distributed MIMO system capacity analysis}

In the case where the origin does not know the channel condition, the best strategy is to transmit the transmitting antennas at equal power. In this case, the MIMO channel capacity is calculated as follows [10-12]:

$$
C=\log _{2} \operatorname{det}\left(I_{n_{\mathrm{R}}}+\frac{P}{\sigma^{2} n_{\mathrm{T}}} H H^{H}\right) \mathrm{bit} / \mathrm{s} / \mathrm{Hz}
$$

where $n_{\mathrm{R}}$ and $n_{\mathrm{T}}$ are the number of receiving and transmitting antennas, respectively; $I_{n_{\mathrm{R}}}$ and $I_{n_{\mathrm{T}}}$ are the $n_{\mathrm{R}} \times$ $n_{\mathrm{R}}$ and $n_{\mathrm{T}} \times n_{\mathrm{T}}$ unit matrices, respectively; $P$ is the total transmitting power; and $m$ is the noise power. For ease of analysis, the channel matrix $H$ is normalized according to Eq. (2), and an average received signal-to-noise ratio is introduced in the capacity calculation formula.

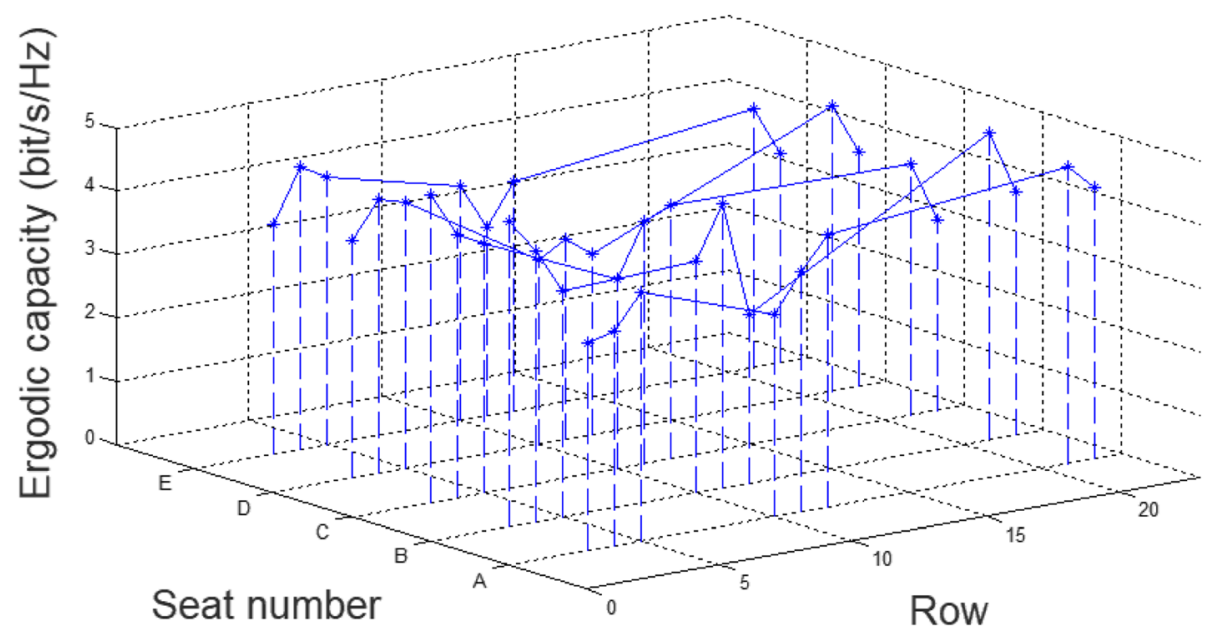

Fig. 1 Distributed $3 \times 3 \mathrm{MIMO}$ measured capacity distribution in the cabin scene, received noise ratio $20 \mathrm{~dB}$ 


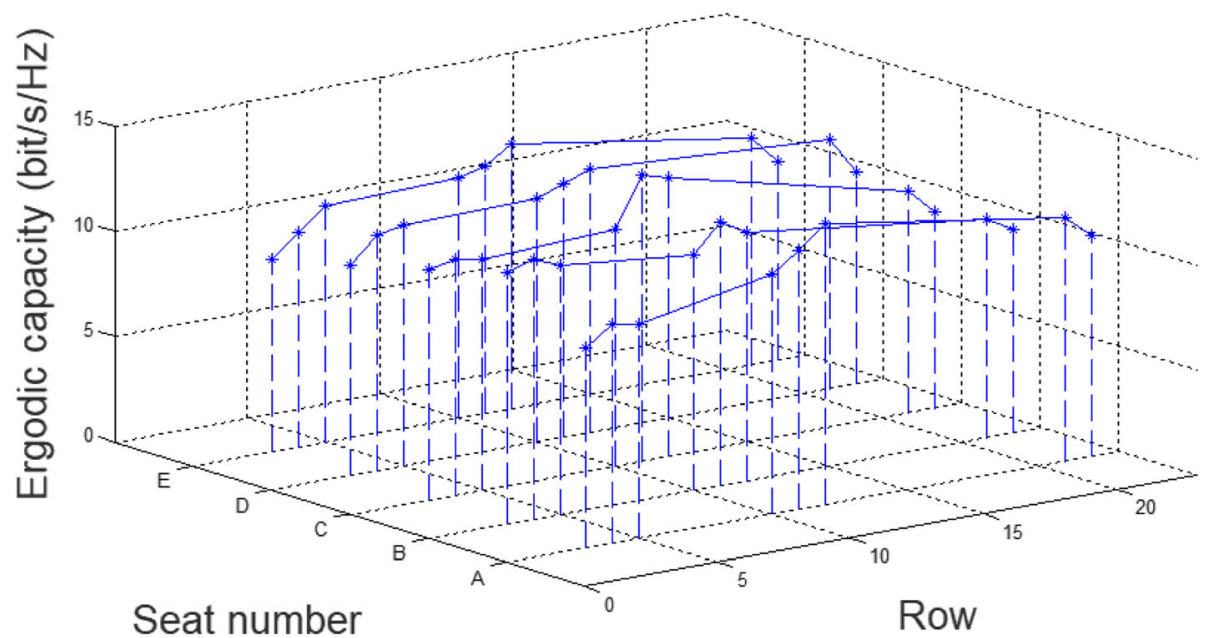

Fig. 2 Distributed $5 \times 5 \mathrm{MIMO}$ measured capacity distribution in the cabin scene, received noise ratio $20 \mathrm{~dB}$

After normalization, the MIMO capacity calculation formula is Eq. (3):

$$
\begin{aligned}
& \left(\sum_{i=1}^{n_{\mathrm{R}}} \sum_{j=1}^{n_{\mathrm{T}}}\left|h_{i j}\right|^{2}\right) / n_{\mathrm{R}} n_{\mathrm{T}}=1 \\
& C=\log _{2} \operatorname{det}\left(I_{n_{\mathrm{R}}}+\frac{\rho}{n_{\mathrm{T}}} \hat{H} \hat{H}^{H}\right) \mathrm{bit} / \mathrm{s} / \mathrm{Hz}
\end{aligned}
$$

In Eq. (3), $\rho$ is the average received signal-to-noise ratio of the $n_{\mathrm{R}}$ receiving antennas, and $\hat{H}$ is the normalized channel matrix. The MIMO channel capacity can also be written in the form of the sum of the equivalent parallel channel capacities:

$$
C=\sum_{i=1}^{m} \log _{2}\left(1+\frac{\rho \lambda_{i}^{2}}{n_{\mathrm{T}}}\right) \mathrm{bit} / \mathrm{s} / \mathrm{Hz}
$$

$\lambda_{1}^{2}, \lambda_{2}^{2}, \ldots \lambda_{m}^{2}$ are the $m$ non-zero eigenvalues of $\hat{H} \hat{H}^{H}$ (the eigenvalues of the matrices $\hat{H} \hat{H}^{H}$ and $\hat{H}^{H} \hat{H}$ are the same).

For wideband frequency selective fading channels, we take the channel capacity as the average of each frequency point $[13,14]$ :

$$
C=\frac{1}{B} \int_{B} \log _{2} \operatorname{det}\left[I_{n_{\mathrm{R}}}+\frac{\rho}{n_{\mathrm{T}}} \hat{H}(f) \hat{H}^{H}(f)\right] \mathrm{dfbit} / \mathrm{s} / \mathrm{Hz}
$$

where $B$ is the channel bandwidth. There are two criteria for evaluating the MIMO channel capacity for random channels: traversal capacity and interrupt capacity. The

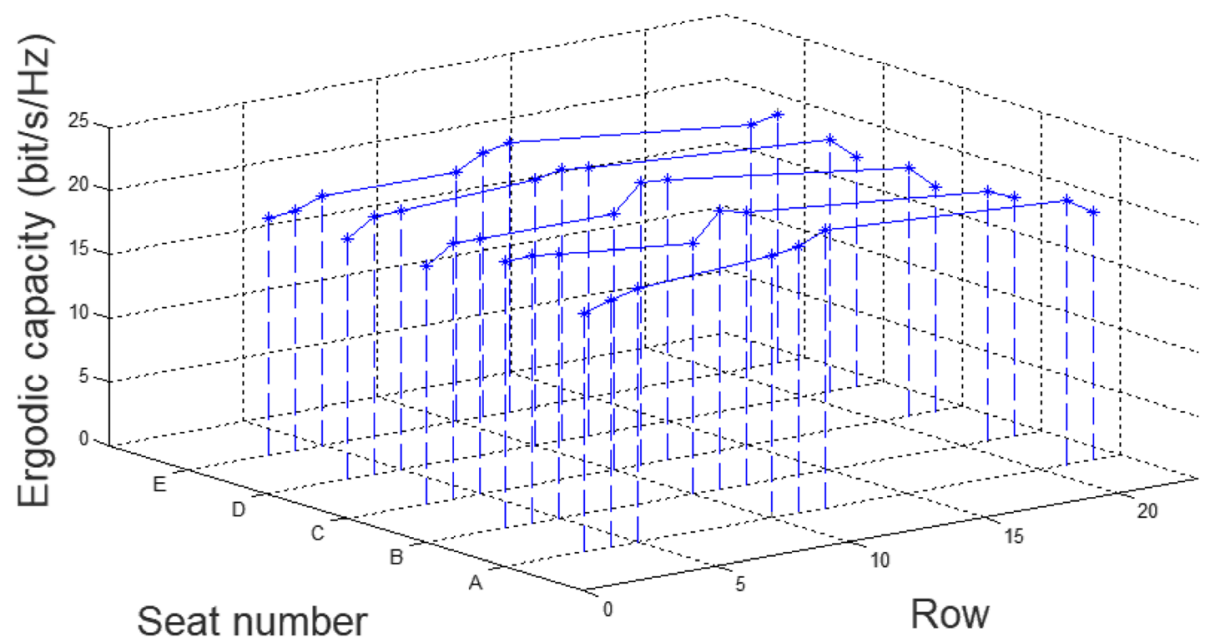

Fig. 3 Distributed $7 \times 7 \mathrm{MIMO}$ measured capacity distribution in the cabin scene, received noise ratio $20 \mathrm{~dB}$ 


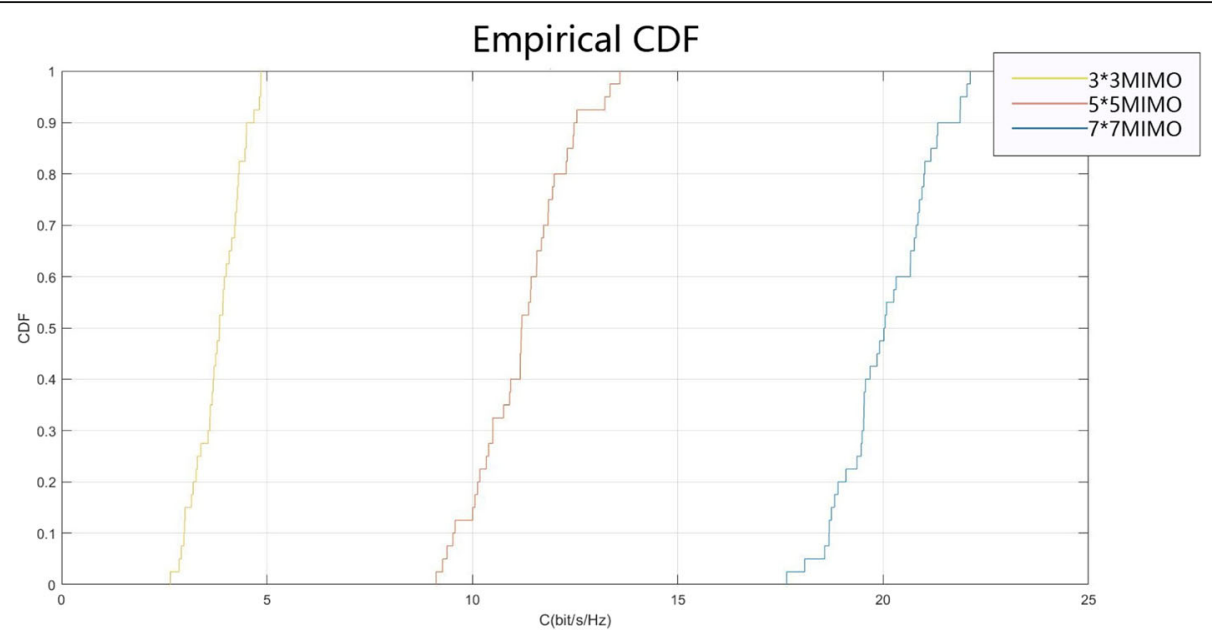

Fig. 4 The different number of antenna channel capacity cumulative probability distribution

ergodic capacity refers to the average channel capacity, and the interrupt capacity can be obtained from the cumulative probability distribution of the capacity.

\section{The effect of different antenna pairs on the channel capacity}

The actual measurement is a $7 \times 7$ distributed MIMO channel matrix, but by selecting different combinations of transmitting and receiving antennas, different MIMO channel matrices can be formed. When one antenna is selected at the transceiver end, a single-transmitting single-receiving system (SISO) is formed. Based on this, the cumulative probability distribution results of the channel capacity for the 3-transmitting-3-receiving, 5-transmitting-5-receiving, and 7-transmitting-7-receiving distributed MIMO systems in the measured scenario are calculated. To ensure fairness, distributed MIMO systems with different configurations of the number of transmitting and receiving antennas in the same scenario have the same total transmitting power and remain fixed $(0 \mathrm{dBm})$ during the test. Each distributed MIMO system consisting of different transmitting and receiving antennas satisfies the following requirements: the transmitting antennas are evenly distributed in the test scenario, and the spacing of the array elements at the receiving end is half a wavelength.

For each system, we calculated the channel capacity at each test location in the cabin using the measured data according to Eq. (5). Figures 1, 2, and 3 show the channel capacity of the three systems, respectively.

It can be seen from the measured results that the distributed MIMO has a significant capacity gain as the number of antennas increases. From the 3-transmitting3-receiving system to the 7-transmitting-7-receiving system, the channel capacity is improved by $3.5 \mathrm{bit} / \mathrm{s} / \mathrm{Hz}$ for

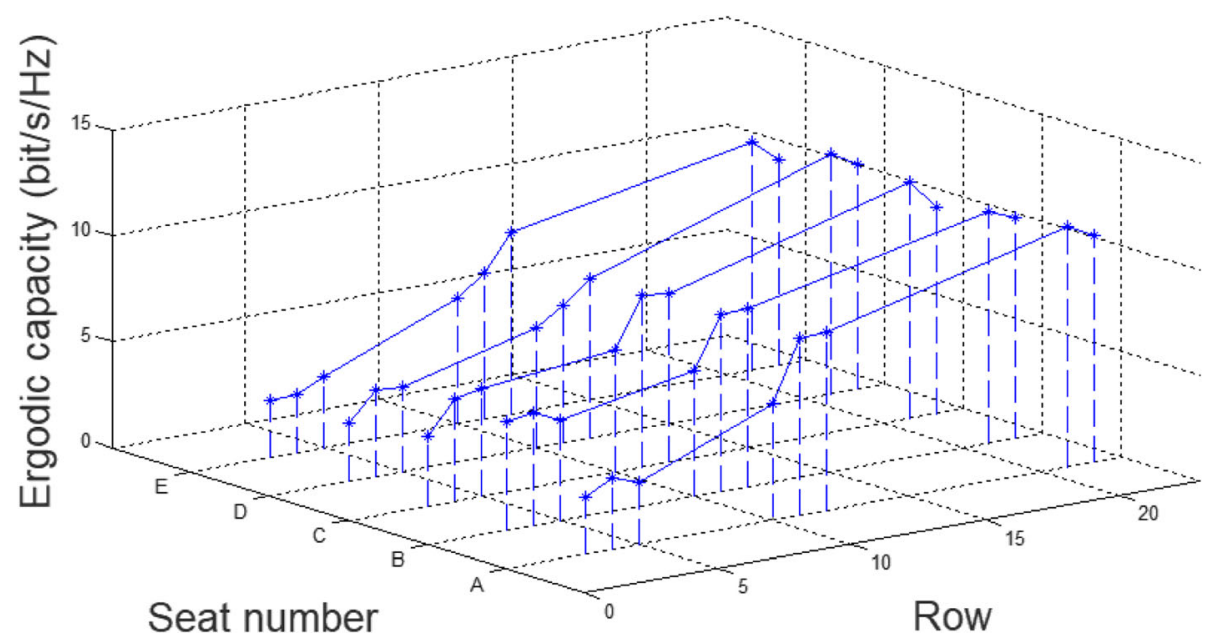

Fig. 5 Distributed $4 \times 4(1,3,5,7)$ MIMO measured capacity distribution in the cabin scene, received noise ratio $20 \mathrm{~dB}$ 


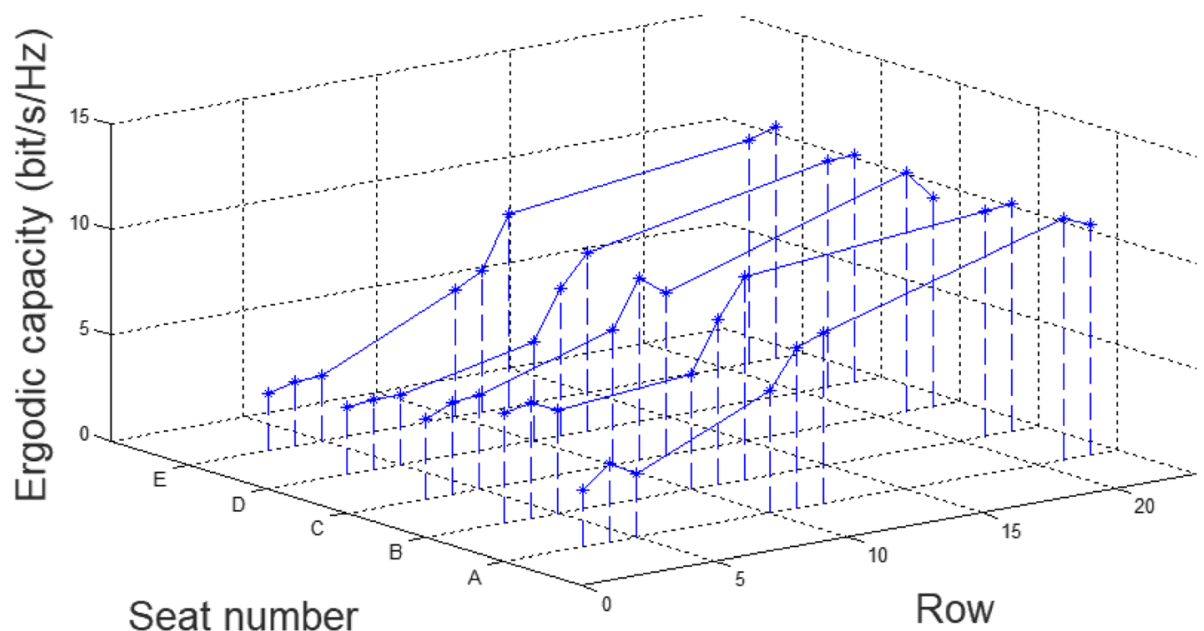

Fig. 6 Distributed $4 \times 4(1,2,3,4) \mathrm{MIMO}$ measured capacity distribution in the cabin scene, received noise ratio $20 \mathrm{~dB}$

each addition of one pair of receiving and transmitting antennas in the distributed MIMO systems.

In Fig. 4, the abscissa indicates the channel capacity in units of bits per second per hertz and the ordinate is the probability of interruption. This indicates that in the case of a distributed antenna placement, the channels of each transmitting antenna arriving at the receiving end are highly irrelevant, and the systems are then able to obtain a large diversity and multiplexing gain.

\section{Influence of different distributions on the channel capacity}

If the antenna is selected for the origin, it is evenly distributed indoors. Here, four antennas are uniformly distributed in the nacelle, namely the first, third, fifth, and seventh antennas, and four other antennas are also selected at the receiving end. The capacity distribution of the static test points in the center at the center frequency is shown in Fig. 5. In order to compare this with the uniform antenna distribution, two sets of non-uniformly distributed transmitting antennas are selected, namely $1,2,3$, and 4 , and 2, 4, 5, and 6 . The channel capacity is as shown in Figs. 6 and 7.

It can be seen that when the antenna is placed sufficiently and uniformly indoors, the distribution of static measuring points, except for the points measured near the origin, will tend to be uniform. When different antenna combinations are selected at the origin, the capacity distribution is as shown in Fig. 8.

It can be seen from Fig. 8 that when the transmitting antennas are combined into $1,3,5$, and 7 , that is, the distribution of the selected transmitting antennas is more uniform than other combinations, the corresponding capacity becomes larger. The further statistics show that the capacity distribution is also more uniform. Therefore, for a distributed antenna system, having the

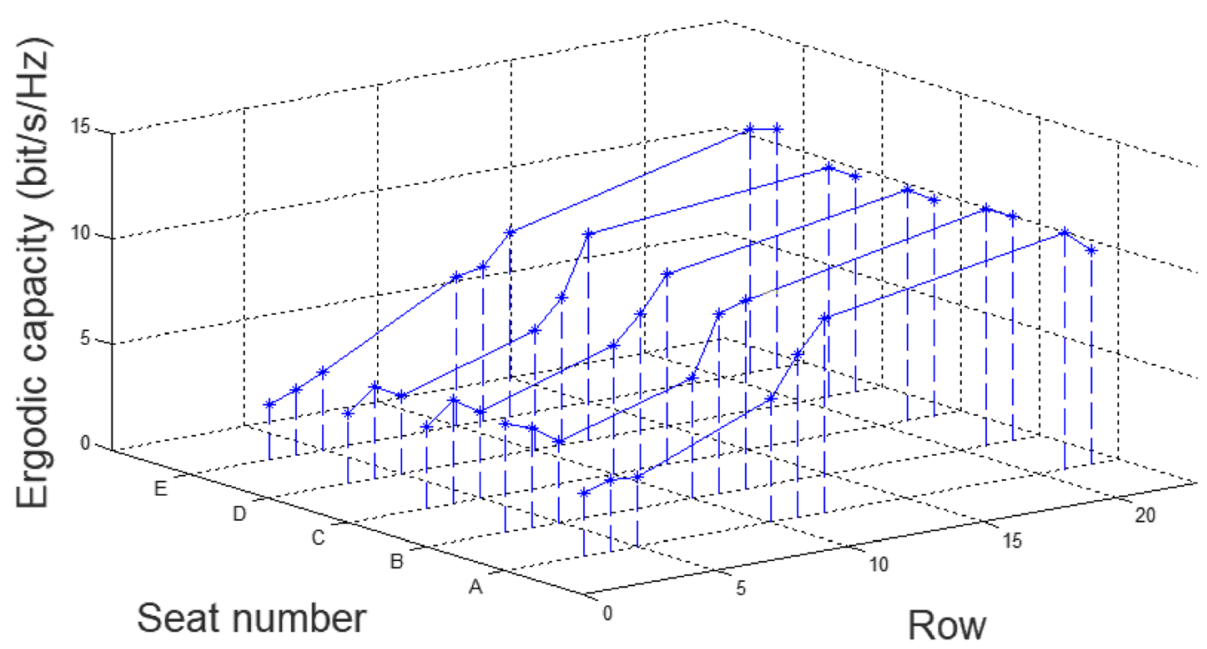

Fig. 7 Distributed $4 \times 4(2,4,5,6)$ MIMO measured capacity distribution in the cabin scene, received noise ratio $20 \mathrm{~dB}$ 


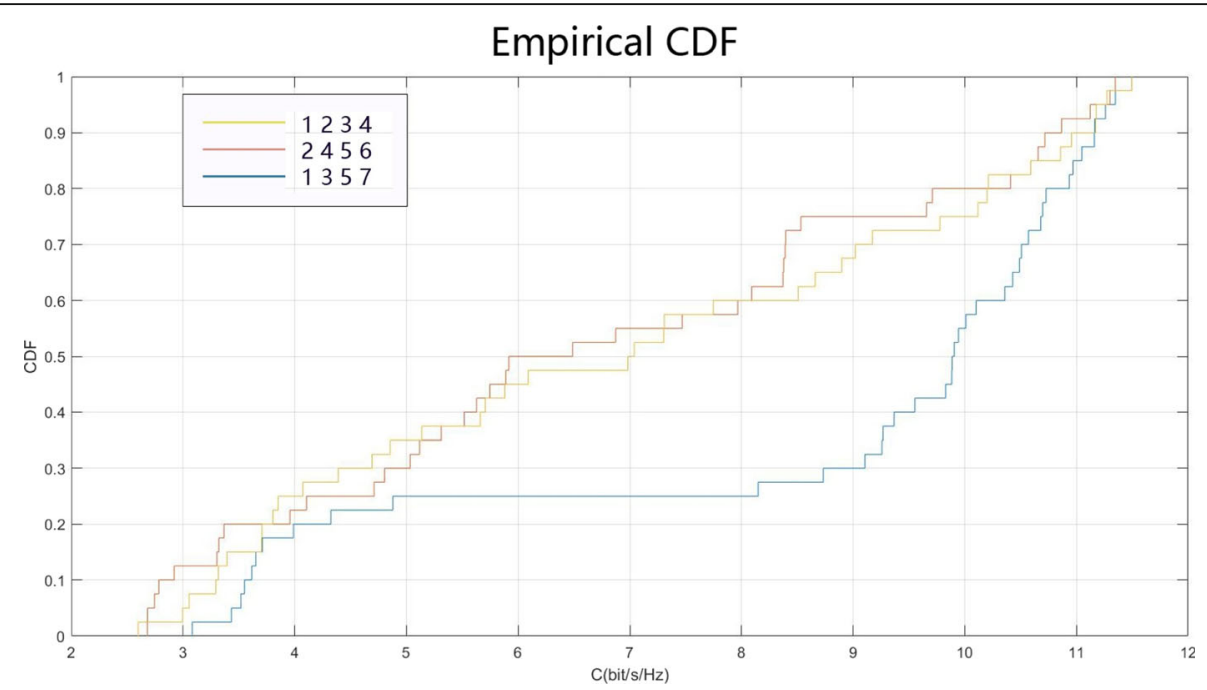

Fig. 8 The same number of antenna under the different distribution of the cumulative probability distribution channel capacity

antennas be evenly distributed is advantageous not only for improving the capacity performance, but also for making the capacity distribution more uniform.

\section{Results and discussion}

The channel measurements of a distributed antenna system were analyzed to determine the cabin capacity characteristics. For a number of fixed-point measurements, the channel at which the transmitting antennas arrive at the receiving end is highly irrelevant in the case of a distributed placement of the transmitting antennas. In this scenario, the channel is approximately equivalent to an independent and identically distributed complex Gaussian channel, and its capacity increases approximately linearly with the number of antennas. At the same time, when the number of antennas is the same but the placement positions are different, the uniformly placed antennas have a larger channel capacity and a more uniform distribution. In short, in the distributed system, the average access distance of the mobile terminal is small, which enhances the coverage performance of the system. Moreover, due to the distributed placement of the antennas, the decoupling characteristics of each channel are good, which greatly improves the capacity performance of the system.

\section{Abbreviations}

5G: The 5th generation wireless systems; MD-82: Douglas MacDonald 82 aircraft; MIMO: Multiple input multiple output; SISO: Single input single output

\section{Acknowledgements}

The authors would like to thank Tsinghua University for providing the measurement data. The first author also would like to thank Professor Chunping Hou and Dr. Qing Wang for the helpful instructions.

\section{Authors' contributions}

$X Z$ conducted the channel model research, participated in the channel capacity analysis, and wrote the manuscript. PZ and QZ did the data processing. YL participated in the study design and statistical analysis. RX and YL participated in its design and coordination and helped draft the manuscript. All authors read and approved the final manuscript. All contributors who do not meet the criteria for authorship should be listed in the "Acknowledgements" section.

\section{Funding}

This work was supported by the Natural Science Foundation of Tianjin City (No. 18JCYBJC86400), Doctor Fund of Tianjin Normal University (No. 52XB1604), Natural Science Foundation of Tianjin City (No. 18JCQNJC70900), National Natural Science Foundation of China (No. 61704122), and National Natural Science Foundation of China (No. 61801327).

Availability of data and materials

Data sharing is not applicable to this article as no datasets were generated or analyzed during the current study.

\section{Competing interests}

The authors declare that they have no competing interests.

\section{Author details}

${ }^{1}$ Tianjin Key Laboratory of Wireless Mobile Communications and Power Transmission, Tianjin Normal University, Tianjin 300387, China. ${ }^{2}$ College of Electronic and Communication Engineering, Tianjin Normal University, Tianjin 300387, China. ${ }^{3}$ International College of Engineers, Tianjin University, Tianjin 300072, China. ${ }^{4}$ Electrical and Information Engineering, Tianjin University, Tianjin 300072, China.

Received: 14 April 2019 Accepted: 11 July 2019

Published online: 23 July 2019

References

1. S. Li, D.X. Li, S. Zhao, 5G internet of things: a survey. J. Ind. Inf. Integr 10, 1-9 (2018)

2. C. Campolo, A. Molinaro, A. lera, et al., $5 \mathrm{G}$ network slicing for vehicle-toeverything services. IEEE Wirel. Commun. 24(6), 38-45 (2018)

3. S. Chen, Q. Fei, H. Bo, et al., User-centric ultra-dense networks for 5G. IEEE Wirel. Commun. 23(2), 78-85 (2018)

4. R. Ibernon-Fernandez, J.M. Molina-Garcia-Pardo, L. Juan-Llacer, Comparison between measurements and simulations of conventional and distributed MIMO system. IEEE Antennas Wirel. Propag. Lett. 7, 546-549 (2008)

5. J. Liang, Q. Liang, Outdoor propagation channel modeling in foliage environment. IEEE Trans. Veh. Technol. 59(5), 2243-2252 (2010)

6. Q. Liang, Radar sensor wireless channel modeling in foliage environment: UWB versus narrowband. IEEE Sensors J. 11(6), 1448-1457 (2011)

7. Z. Yan, L. Zhenghui, L. Fengyu, et al., Measurement-based analysis of transmit antenna selection for in-cabin distributed MIMO system. Int. J. Antennas Propag. 2012, 1-6 (2012) 
8. Xiaonan Z, Chunping H, Qing W, A new SVM-based modeling method of cabin path loss prediction. Int. J. Antennas Propag. 2013, 1-7 (2013)

9. X. Zhao, C. Hou, Q. Wang, et al., Location refinement and power coverage analysis based on distributed antenna. Trans. Tianjin Univ. 22(1), 7-10 (2016)

10. Li Z, Luan F, Zhang Y, et al. Capacity and spatial correlation measurements for wideband distributed MIMO channel in aircraft cabin environment. Wireless Communications \& Networking Conference. (IEEE, Shanghai, 2012). https://doi.org/10.1109/WCNC.2012.6213954

11. A.V. Reznichenko, I.S. Terekhov, in Information Theory Workshop. Channel capacity and simple correlators for nonlinear communication channel at large SNR and small dispersion (2018)

12. Khalili A, Rini $S$, Barletta $L$, et al. On MIMO channel capacity with output quantization constraints. 2018

13. R.D. Vieira, J.C.B. Brandao, G.L. Siqueira, in International Telecommunications Symposium. MIMO measured channels: capacity results and analysis of channel parameters (2008)

14. A.F. Molisch, M. Steinbauer, M. Toeltsch, et al., Capacity of MIMO systems based on measured wireless channels. IEEE J. Sel. Areas Commun. 20(3), 561-569 (2006)

\section{Publisher's Note}

Springer Nature remains neutral with regard to jurisdictional claims in published maps and institutional affiliations.

\section{Submit your manuscript to a SpringerOpen ${ }^{\circ}$ journal and benefit from:}

- Convenient online submission

- Rigorous peer review

- Open access: articles freely available online

High visibility within the field

- Retaining the copyright to your article

Submit your next manuscript at $\boldsymbol{\nabla}$ springeropen.com 